\title{
Smagorinsky constant in LES modeling of anisotropic MHD turbulence
}

Received: 3 May 2006 / Accepted: 7 August 2007

(C) Springer-Verlag 2007

\begin{abstract}
Turbulent fluctuations in magnetohydrodynamic (MHD) flows can become strongly anisotropic or even quasi-2D under the action of an applied magnetic field. We investigate this phenomenon in the case of low magnetic Reynolds numbers. It has been found in earlier DNS and LES of homogeneous turbulence that the degree of anisotropy is predominantly determined by the value of the magnetic interaction parameter and only slightly depends on the Reynolds number, type of large-scale dynamics, and the length scale. Furthermore, it has been demonstrated that the dynamic Smagorinsky model is capable of self-adjustment to the effects of anisotropy. In this paper, we capitalize on these results and propose a simple and effective generalization of the traditional non-dynamic Smagorinsky model to the case of anisotropic MHD turbulence.
\end{abstract}

Keywords Magnetohydrodynamic Turbulence $\cdot$ Large-Eddy Simulation

PACS 47.65.-d · 47.27.ep

\section{Introduction}

Magnetohydrodynamic (MHD) turbulent flows occur in numerous astrophysical, geophysical, and technological applications. In this paper, we consider the case of low magnetic Reynolds number

$$
R_{m} \equiv u L / \eta \ll 1,
$$

typical for the industrial and laboratory flows of liquid metals, oxide melts, and other electrically conducting fluids (see, e.g., [1] or [2]). In (1), $u$ and $L$ are the typical velocity and length scales, and $\eta=\left(\sigma \mu_{0}\right)^{-1}$ is the magnetic diffusivity; $\sigma$ and $\mu_{0}$ are the electric conductivity of the liquid and the magnetic permeability of vacuum.

Low- $R_{m}$ interaction between a static magnetic field and a turbulent flow is an important factor of some metallurgical operations, such as continuous steel casting or growth of large semiconductor crystals, where magnetic fields are intentionally used to non-intrusively suppress the unwanted development of the flow. In other cases, such as the primary aluminum production in Hall-Héroult or inert anode processes, or in the lithium cooling blankets for magnetic confinement fusion, the inevitably present static magnetic field has an adverse effect on performance, which has to be minimized through optimization of the process.

The results of the present work, albeit rigorously valid only in the case of low $R_{m}$, can be extended to the situations with moderate $R_{m}$ and high hydrodynamic Reynolds number, most notably to the earth dynamo problem. The diffusive cut-off scale of the magnetic field is sufficiently large in such cases so that the magnetic

Communicated by R.D. Moser

A. Vorobev · O. Zikanov ( $\varangle)$

Department of Mechanical Engineering, University of Michigan-Dearborn, Dearborn, MI 98128-1491, USA

E-mail: zikanov@umd.umich.edu 
field can be fully resolved in the DNS-like manner. The task of modeling the turbulent velocity fluctuations at smaller scales reduces to the problem addressed in this paper.

We will consider incompressible flows and, for simplicity, assume that the applied magnetic field is uniform and vertical $\mathbf{B}=B \mathbf{e}_{z}$. In the low- $R_{m}$ case, the MHD equations can be significantly simplified by applying the quasi-static approximation [2]. The perturbations of the magnetic field induced by fluid motions are small in comparison with the imposed magnetic field and can be neglected. They can also be approximately assumed to adjust instantaneously to the velocity perturbations. In the result of these approximations, the Lorentz force is expressed as a linear functional of velocity. The governing equations can be represented in a closed form as

$$
\partial_{t} \mathbf{u}+(\mathbf{u} \cdot \nabla) \mathbf{u}=-\rho^{-1} \nabla p+v \Delta \mathbf{u}-\sigma B^{2} \rho^{-1} \Delta^{-1} \partial_{z z} \mathbf{u}, \quad \nabla \cdot \mathbf{u}=0,
$$

where $\Delta^{-1}$ is the reciprocal Laplace operator that stands for a solution of the Poisson equation for the electric potential with proper boundary conditions. The non-dimensional form of (2) contains two dimensionless parameters, one of which is the Reynolds number $R e \equiv u L / v$ and another is the magnetic interaction parameter $N \equiv \sigma B^{2} L / \rho u$ that estimates the ratio between the Lorentz and inertia forces.

We focus on MHD turbulence far from the walls and consider the implications of the special properties resulting from the presence of the Lorentz force term in (2) for large-eddy simulations. Such properties have been relatively thoroughly studied in analytical, experimental, and numerical works (see, for example, [3-6]). It has been understood that in unbounded flows the direct effect of the magnetic field is twofold. First, there is an additional turbulence suppression via Joule dissipation of induced electric currents. Second, the flow acquires axisymmetric anisotropy with flow structures elongated in the direction of the magnetic field. The nature of the anisotropy becomes transparent if we use the Fourier transform to write the rate of the Joule dissipation of a mode $\widehat{\mathbf{u}}(\mathbf{k}, t)$ as

$$
\mu(\mathbf{k})=\sigma B^{2} \rho^{-1}|\widehat{\mathbf{u}}(\mathbf{k}, t)|^{2} \cos ^{2} \theta,
$$

where $\theta$ is the angle between the wavenumber vector $\mathbf{k}$ and the magnetic field $\mathbf{B}$. The Joule dissipation tends to eliminate the velocity gradients along the magnetic field lines, thus leading to elongation of flow structures. The flow approaches 2D form with zero parallel gradients at $N \rightarrow \infty$, although it has been argued in [7] that the proper term is "quasi-two-dimensionality" due to the inevitable elliptic and shear flow instabilities of the 2D structures. Furthermore, pure two-dimensionality is impossible in the presence of walls non-parallel to the magnetic field (see, e.g., [8]).

Only the anisotropy of gradients is directly affected by the magnetic field. Another type of anisotropy referring to inequality between the velocity components (anisotropy of the Reynolds stress tensor) can follow from the action of the magnetic field indirectly, through the nonlinear interaction mechanism. Other indirect effects include the suppression of the nonlinear energy transfer between the length scales and the associated increase of the inertial range slope of the energy power spectrum (approaching $k^{-3}$ in quasi-2D flows at high $N$ ).

The focus of the present paper is on the efficient LES modeling of anisotropic MHD turbulence. The term "efficiency" is understood in a broader sense to include the model's accuracy, breadth of applicability, and low computational cost. More precisely, we would like to develop a model that could be applied to MHD flows with

- Accuracy comparable with the accuracy achieved for non-magnetic flows at similar $R e$ and numerical resolution.

- Applicability range $0 \leq N<\infty$, i.e., for the entire spectrum of MHD flows, from nearly isotropic to quasi-2D.

- Lowest possible computational cost, preferably not exceeding the computational cost of models commonly used in the non-magnetic case.

In the next section, we discuss the properties of the anisotropic MHD turbulence and review the earlier modeling attempts. The discussion serves as a basis for Sect. 3, where we investigate the possibility of a simple generalization of the Smagorinsky closure model to the MHD case by assuming a functional dependency of the Smagorinsky constant on a single anisotropy parameter. The final remarks are given in Sect. 4.

\section{Modeling of anisotropic MHD turbulence: results of earlier studies}

A detailed study of the anisotropy of homogeneous MHD turbulence at low $R_{m}$ was conducted in [6]. DNS and LES computations were performed in a wide range of $R e$ and $N$. In order to achieve a statistically steady flow, 
the artificial force $\hat{\mathbf{f}}(\mathbf{k})=\alpha(\mathbf{k}) \hat{\mathbf{u}}(\mathbf{k})$ was applied to the large scale modes with $1.5 \leq k \leq 3.1$. The coefficients $\alpha$ were determined at each time step so as to provide the total work by forcing equal to a prescribed constant $\epsilon_{0}$ according to $\alpha(\mathbf{k})=\epsilon_{0} /\left(N_{\text {forced }}|\hat{\mathbf{u}}(\mathbf{k})|^{2}\right)$, where $N_{\text {forced }}$ is the number of the forced modes. This led to statistically steady states, in which the total (viscous plus magnetic) dissipation rate fluctuated only slightly around $\epsilon_{0}$. In order to reveal the possible effect of the large-scale dynamics, two types of forcing were used, one isotropic with the energy input equally divided among the forced modes and another purely $2 \mathrm{D}$ with the energy input limited to the modes with $\mathbf{k} \perp \mathbf{B}$. Below we identify these two types as $3 \mathrm{D}$ and $2 \mathrm{D}$ forcing, respectively.

The main results of [6] deal with the transformation of turbulent fluctuations at intermediate and small length scales. The scale-dependent gradient anisotropy was estimated using

$$
g(k) \equiv \frac{3 \tau}{2} \frac{\mu(k)}{E(k)}=\frac{3 \sum \frac{k_{z}^{2}}{k^{2}} \hat{\mathbf{u}} \cdot \hat{\mathbf{u}}^{*}}{\sum \hat{\mathbf{u}} \cdot \hat{\mathbf{u}}^{*}},
$$

where the sums are over all wavenumber vectors in the shell $k-1 / 2<|\mathbf{k}| \leq k+1 / 2$, and $\mu(k), E(k)$ are the power spectra of the magnetic dissipation rate and kinetic energy. The scaling factor in (4) uses the Joule damping time $\tau \equiv \rho / \sigma B^{2}$ and is chosen so that $g(k)=1$ in an isotropic flow and $g(k)=0$ in a purely 2D flow with zero magnetic dissipation.

It has been found that at all $k$ outside the range of artificial forcing, $g(k)$ is a remarkably robust function of the magnetic interaction parameter $N$. The influence of the length scale, $R e$, and the details of the largescale dynamics (dictated by the different types of the forcing) is much weaker. Similar, although slightly less pronounced behavior was observed for the scale-related anisotropy of velocity components.

Another result of [6] important for the present discussion concerns the meaning of the coefficients

$$
G_{i j}=\frac{\left\langle\left(\partial u_{i} / \partial z\right)^{2}\right\rangle\left(1+\delta_{i 3}\right)}{\left\langle\left(\partial u_{i} / \partial x_{j}\right)^{2}\right\rangle\left(1+\delta_{i j}\right)}, \quad i=1,2,3, j=1,2,
$$

where $\langle\cdots\rangle$ stands for the volume averaging. Averaging over the entire computational domain was applied in [6] following the assumption of spatial homogeneity. It should be replaced by some local averaging in the inhomogeneous flows.

The coefficients (5) are equal to one in an isotropic flow (see [9]) and zero in a purely 2D flow uniform in the direction of the magnetic field, and are usually close to each other in anisotropic flows so that any of them can be used to evaluate the anisotropy. In our study, $G_{22}$ and $G_{32}$ are calculated and averaged to a single coefficient $G=\left(G_{22}+G_{32}\right) / 2$. Formally, $G$ is an integral characteristic of the gradient anisotropy. One could think of it as related to the large energy-containing scales of the flow. In fact, however, it was demonstrated in [6] that the dominant contribution into $G$ is from the intermediate length scales smaller than those where the forcing is applied but larger than the typical scale of LES cut-off. As a result, $G$ was found to be close to the scale-dependent anisotropy coefficient $g(k)$ in the range of its scale invariance and insensitive to the details of large-scale dynamics, Reynolds number, and the use of LES models. $G$ can be considered as a reasonably accurate measure of anisotropy in the intermediate scale range.

LES modeling of decaying and forced homogeneous MHD turbulence was conducted in [10] and [6], respectively. The Smagorinsky eddy viscosity closure hypothesis was used in both cases with the subgridscale stress tensor $\bar{\tau}_{i j}$ expressed through the rate-of-strain tensor of filtered velocity field $\bar{S}_{i j}$ as

$$
\bar{\tau}_{i j}=-2 C_{S} \bar{\Delta}^{2}|\bar{S}| \bar{S}_{i j}, \quad|\bar{S}|=\left(2 \bar{S}_{i j} \bar{S}_{i j}\right)^{1 / 2},
$$

where $\bar{\Delta}$ is the filter width and $C_{S}$ is the Smagorinsky constant. The conclusion of both studies, achieved through the a-posteriori comparison with the results of high resolution DNS, was that the Smagorinsky model is overdissipative if used in its simple form with constant $C_{S}$. Substantially better agreement with the DNS results was achieved when the constant $C_{S}$ was determined using the dynamic procedure $[11,12]$. In the procedure, a second "test" filter with the width $\widetilde{\Delta}>\bar{\Delta}$ is applied to the computed flow field. It is assumed that the relation (6) holds for both the main and the test length scales. This leads to the formula for determining the Smagorinsky constant based on the flow conditions [12]

$$
C_{S}=\left\langle\widetilde{\bar{M}}_{i j} \widetilde{\bar{L}}_{i j}\right\rangle /\left\langle\widetilde{\bar{M}}_{i j} \tilde{\bar{M}}_{i j}\right\rangle,
$$

where $\widetilde{\bar{L}}_{i j}=\widetilde{\overline{\bar{u}}_{i} \bar{u}_{j}}-\widetilde{\bar{u}}_{i} \widetilde{\bar{u}}_{j}$ is the Leonard tensor, and $\widetilde{\bar{M}}_{i j}=2\left[\bar{\Delta}^{2}\left|{\widetilde{\bar{S}} \mid \bar{S}_{i j}}-\widetilde{\Delta}^{2}\right| \widetilde{\bar{S}}_{\bar{S}} \widetilde{\bar{S}}_{i j}\right]$. 
The fact that the simple Smagorinsky model with constant $C_{S}$ is inadequate in the case of MHD flows with high $N$ is quite expectable. The values used for $C_{S}$ (between 0.01 and 0.0324 depending on the type and width of the filter and the strength of mean shear [13]) do not take into account the flow transformation caused by the applied magnetic field (anisotropy, suppressed nonlinear energy transfer, and steepened energy spectrum). All these factors lead to reduction of the subgrid-scale energy dissipation and, thus, require reduction of $C_{S}$.

The better accuracy of the dynamic model is, too, not entirely surprising. The model has demonstrated its ability to remedy the overdissipation problem in other situations characterized by reduced SGS dissipation, for example in the laminar-turbulent transition. The physical and mathematical reasons for the accuracy are, however, unclear. Albeit the formula (7) can be derived from the assumption of scale similarity, the assumption itself is invalid in many cases where the dynamic model demonstrated its superiority (for example in the transitional flows or flows at moderate Reynolds numbers). In general, the a-posteriori accuracy of the model can not be convincingly related to its accurate representation of any particular aspect of the behavior of subgrid-scale fluctuations [14]. An alternative, clearly not exhaustive explanation was proposed in [15], where the dynamic procedure was shown to minimize the dependency of modeled quantities on the filter width.

For these reasons, we refrain from attributing the accuracy of the dynamic model in the MHD case to some physical features of the flow (although it can be argued that the scale invariance of the anisotropy is relevant). Instead, the next section presents an attempt to capitalize on the demonstrated ability of the dynamic model to modify $C_{S}$ in accordance to the flow transformation. We try to develop a simple formula for $C_{S}$ that would mimic the dynamic modification.

It can be argued that the isotropic eddy viscosity relation (6) is itself invalid in the case of a strongly anisotropic MHD flow since, as was demonstrated in [16], the anisotropies of the tensors $\bar{\tau}_{i j}$ and $\bar{S}_{i j}$ are very different. We tested several generalizations of the dynamic Smagorinsky model based on the eddy viscosity formulas with one or two dynamically adjustable anisotropy corrections. None of them showed discernibly better agreement with the DNS results than the conventional dynamic model. It seems that simple reduction of $C_{S}$ is sufficient to account for the effect of the imposed magnetic field.

\section{Smagorinsky constant as a function of anisotropy parameters}

For our purposes, the discussion of the previous section can be reduced to the following two hypotheses, which, although not fully proven, received substantial factual support in computations [10] and [6].

1. The dynamic adjustment of $C_{S}$ is sufficient to account for anisotropy and other aspects of the flow transformation caused by the imposed magnetic field. No other modification of the Smagorinsky closure is needed to keep the accuracy of the model at the same level as in the isotropic case.

2. The variation of $C_{S}$ caused by the magnetic field can be adequately described by a function of a single scalar parameter, such as the anisotropy coefficient (5) or the magnetic interaction parameter $N$.

In this section we try to capitalize on the hypotheses and obtain the relations $C_{S}=C_{S}(N)$ and $C_{S}=C_{S}(G)$. One can speculate on which of the two dependencies, $C_{S}(N)$ or $C_{S}(G)$, is preferable. There is no strong preference in the case of homogeneous turbulence, where, as shown in [6] and confirmed by our computations discussed below, $G$ can approximately be considered a one-to-one function of $N$. In the real flows, where $N$ is evaluated based on the size of the flow domain and some constant characteristic velocity, its effective value can change in space (for example because of variation of $\mathbf{B}$ ) or time (for example in the case of decaying flow). The relation $C_{S}=C_{S}(G)$ seems, therefore, preferable as one based on a universal anisotropy characteristic that can be evaluated locally, both in space and time.

Below we present the results of a series of LES computations of homogeneous MHD turbulence in a box of dimensions $2 \pi \times 2 \pi \times 4 \pi$ with periodic boundary conditions. The dynamic Smagorinsky model is used as a subgrid-scale closure. The numerical method is pseudo-spectral based on the fully de-aliased fast Fourier transform. The flow is artificially forced at $1.5 \leq k \leq 3.1$ with the 3D and 2D types of forcing described in the beginning of the previous section (see [6] for more details). Each numerical experiment starts with non-magnetic calculations that last long enough to produce a fully developed turbulent flow. Then, at $t=t_{0}$, the magnetic field $B$ is applied and kept constant till the flow transformation is complete, after which the flow statistics are collected and averaged over several (at least, 3 ) eddy turnover times $T=L\left(t_{0}\right) / u\left(t_{0}\right)$. The Reynolds number $R e$ and the magnetic interaction parameter $N$ are evaluated in the isotropic flow at $t=t_{0}$ using the integral length scale $L$ and the rms velocity $u$. The experiments are conducted at $0 \leq N \leq 10$ and $R e=700,2,500$, and 6,500. Two numerical resolutions with $64 \times 64 \times 128$ and $32 \times 32 \times 64$ spectral 

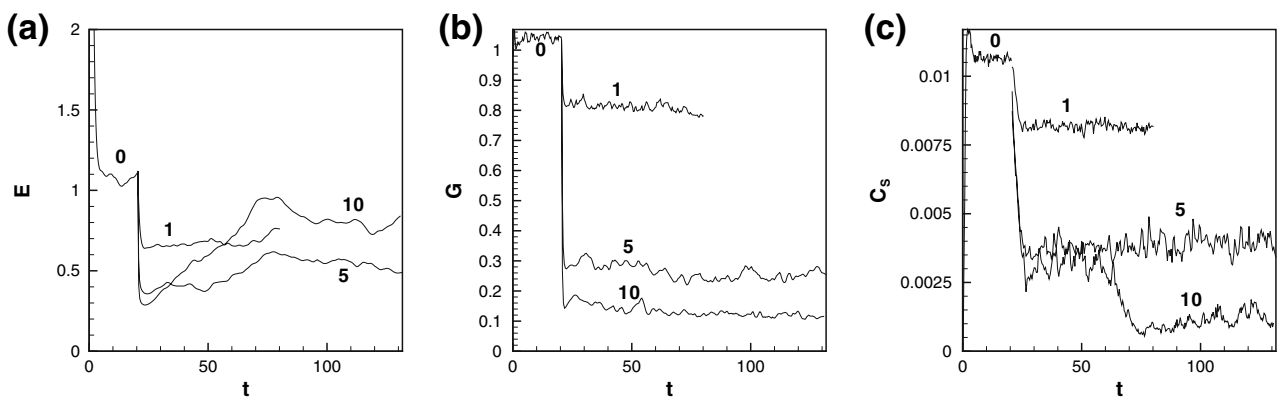

Fig. 1 Flows with 3D forcing, $R e=2,500$, and numerical resolution $64 \times 64 \times 128$. Time evolutions of the resolved total energy a, global anisotropy coefficient $\mathbf{b}$, and volume-averaged Smagorinsky coefficient $\mathbf{c}$ are shown for $N=0,1,5$, and 10
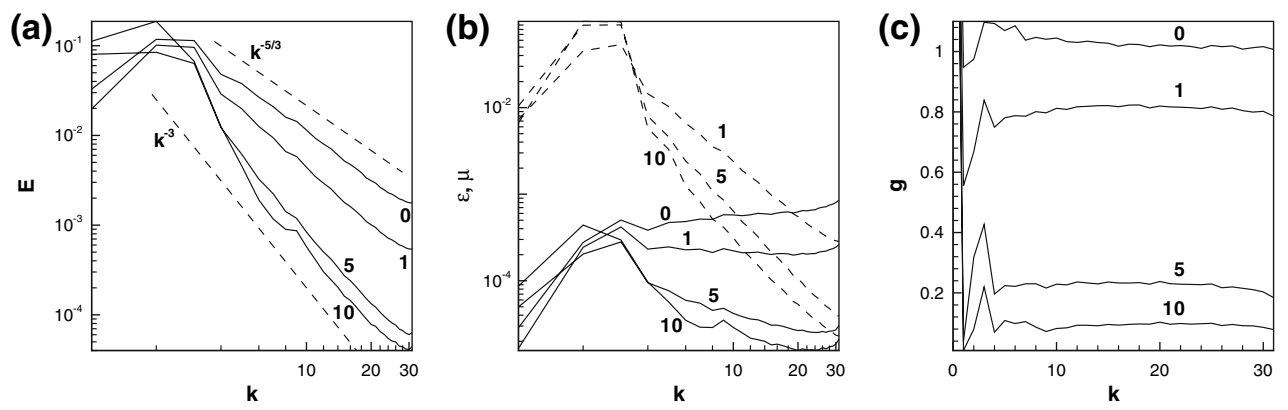

Fig. 2 Flows with 3D forcing, $R e=2,500$, and numerical resolution $64 \times 64 \times 128$. Time averaged spectra of energy a, viscous (solid line) and magnetic (dashed line) dissipation rates $\mathbf{b}$, and the local anisotropy coefficient (4) are shown for $N=0,1,5$, and 10

functions are used. The filtering width $\bar{\Delta}$ of the LES closure is defined as the grid step. Further details of the computational procedure can be found in [6].

Figure 1 illustrates the typical temporal evolution of the flows. The results obtained with 3D forcing at $R e=2,500$ and $N=0,1,5$, and 10 with the numerical resolution of $64 \times 64 \times 128$ are shown. After the introduction of the magnetic field, the global characteristics such as the total resolved energy, rate of resolved viscous dissipation or the anisotropy coefficient $G$ evolve rapidly until they stabilize at new levels corresponding to the forced anisotropic flows. The Smagorinsky constant evolves in the same way but, as can be seen from comparison between the Fig. 1a-c, its evolution to new levels is slower.

Power spectra of the flow energy $E(k)$, resolved viscous $\epsilon(k)$ and magnetic $\mu(k)$ dissipation rates were obtained at the equilibrium stages of each flow. Several flow fields separated by more than one isotropic eddy turnover time from each other were used for the time-averaged spectra, examples of which are presented in Fig. 2. One can see how the increase in the strength of the magnetic field (growth of $N$ ) leads to steeper curves $E(k)$ and $\mu(k)$ and reduction of viscous dissipation at small scales. The scale-dependent anisotropy coefficient $g(k)$ demonstrates the behavior observed and analyzed in [6], i.e., scale-independence outside the forced range. At the strongest magnetic field (at $N=10$ ) the flow becomes strongly anisotropic but not purely 2D as shown by small but non-zero values of $g(k)$ and non-vanishing magnetic dissipation.

Figure 3 shows the effect of the magnetic field on the resolved viscous and magnetic dissipation rates $\epsilon$ and $\mu$ and the subgrid-scale dissipation rate $\epsilon_{S G S}$ in fully developed flows. Time- and volume-averaged rates obtained at different $R e$, types of forcing, and numerical resolution are shown. The numerical experiments are staged in such a way that the total dissipation rate is constant, i.e., $\epsilon+\mu+\epsilon_{S G S}=\epsilon_{0}=0.5$. One can see that at relatively large Reynolds numbers used in the simulations, the resolved scales are responsible for only a small fraction of the total viscous dissipation. $\epsilon$ is less than $0.16 \epsilon_{0}$ at zero magnetic field and decreases with $N$. For the non-magnetic runs, the dissipation is caused predominantly by the Smagorinsky term. In the presence of the magnetic field, $\mu$ increases with $N$ and $\epsilon_{S G S}$ decreases, respectively, although it never drops below $0.1 \epsilon_{0}$.

It is interesting that the role played by the SGS model grows if the 2D forcing is applied (see Fig. 3c). The reasons for that are our requirement of constant total dissipation and the strong anisotropy induced by the 

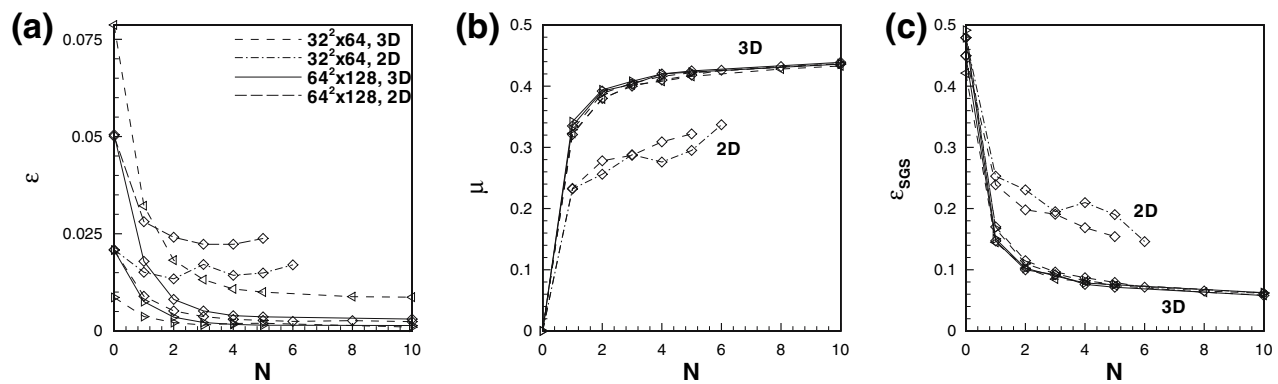

Fig. 3 Resolved viscous $\mathbf{a}$, magnetic $\mathbf{b}$ and subgrid-scale $\mathbf{c}$ dissipation rates as functions of magnetic interaction parameter. Timeand volume-averaged data obtained in fully established flows with 3D and 2D forcing at two different numerical resolutions are shown. Right triangle $R e=700$, diamond $R e=2,500$, left triangle $R e=6,500$
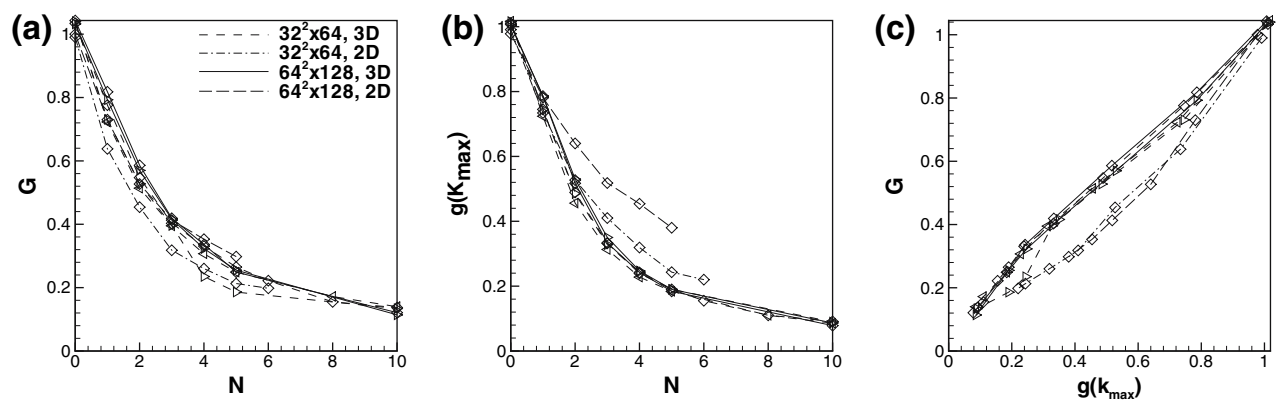

Fig. 4 Global anisotropy coefficient $\mathbf{a}$ and the scale-dependent anisotropy coefficient taken at the scale of filter width $\mathbf{b}$ as functions of magnetic interaction parameter; c global versus filter-width coefficients. Notation is as in Fig. 3

forcing at the large length scales. This results in decrease of the magnetic dissipation rate at these scales and so of the total dissipation rate $\mu$ leading to the growth of $\epsilon_{S G S}$.

The flow anisotropy was estimated using the global coefficient $G$ and the scale-dependent coefficient $g\left(k_{\max }\right)$ taken at the length scale of the filter width. Again, time-averaging over several eddy turnover times was applied. One can see in Fig. $4 \mathrm{a}, \mathrm{b}$ that both coefficients decrease rapidly at small and moderate $N$ and somewhat slower at stronger fields with $N>3$. The flow becomes strongly anisotropic but remains essentially 3D.

An important feature of curves in Fig. 4a, b is their closeness to each other. This is quite remarkable considering the fact that the curves are obtained for substantially different Reynolds numbers, forcing mechanisms, and numerical resolutions. Moreover, as can be seen in Fig. $4 \mathrm{c}, G$ is nearly equal to $g\left(k_{\max }\right)$ and, thus, to coefficients $g(k)$ at any other $k$ outside the range of forced length scales (see Fig. 2c). Our calculations confirm the conclusions obtained in [6] on the basis of the DNS and LES computations at lower Reynolds numbers. The anisotropy properties of the turbulent fluctuations are nearly scale-independent, insensitive to the large scale dynamics and the magnitude of the Reynolds number and are determined by the magnetic interaction parameter $N$ or another parameter, such as the integral anisotropy coefficient $G$.

The main results are presented in Fig. 5, which shows the dependence of the volume- and time-averaged Smagorinsky constant $C_{S}$ on the anisotropy characteristics. As in the previous figures, data obtained at different Reynolds numbers, forcing mechanisms, and numerical resolutions are plotted. The constant decreases with the strength of the magnetic field as represented by increasing $N$ or decreasing anisotropy coefficients.

The results support the second of our hypotheses. The curves in Fig. 5 are quite close to each other. The Smagorinsky constant can be considered a function of $N, G$, or $g\left(k_{\max }\right)$ with a reasonable degree of accuracy. One can see in Fig. 5b that the agreement is particularly good for the function $C_{S}=C_{S}(G)$. Requiring that $C_{S}$ attains its isotropic value at $G=1$ and zero in a purely 2D flow at $G=0$ we can approximate the data in Fig. 5 b by a simple linear relation

$$
C_{S}=C_{S 0} G
$$

shown by the bold line. Here $C_{S 0}$ is the Smagorinsky constant corresponding to the isotropic non-magnetic flow. It is about 0.011 in our calculations but can have different values in other cases depending on type of the flow and type and width of the filter. 

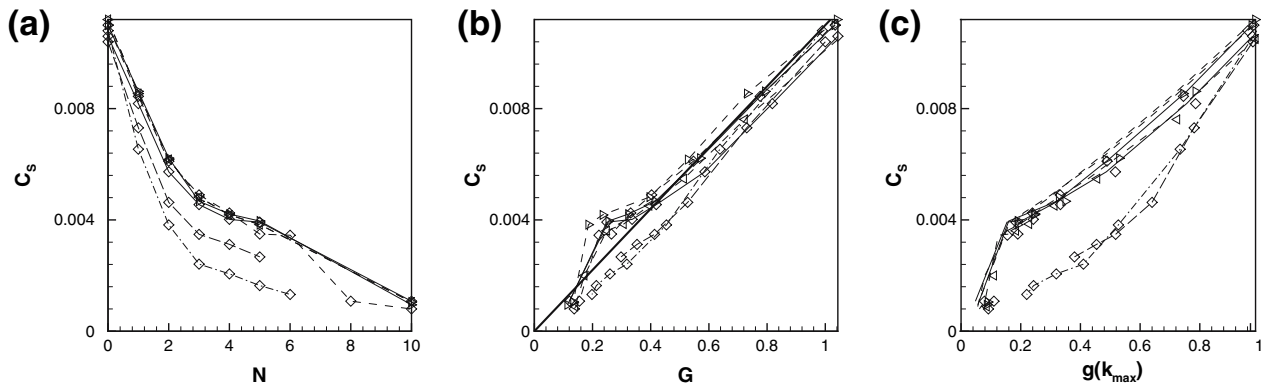

Fig. 5 a Volume- and time-averaged Smagorinsky constant $C_{S}$ as a function of the magnetic interaction parameter a, global anisotropy coefficient $\mathbf{b}$ and coefficient of anisotropy at filter width $\mathbf{c}$. Bold line in $\mathbf{b}$ is for the linear relation (8). Notations are as in Fig. 3
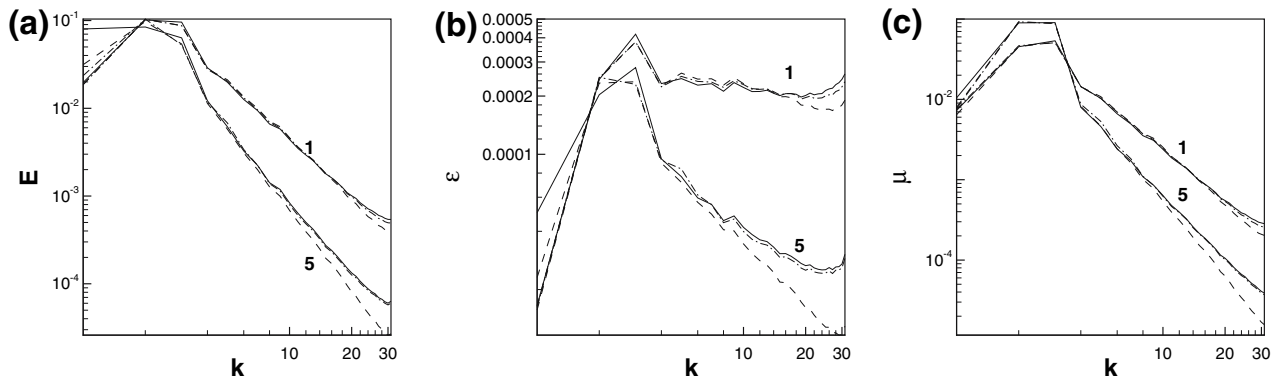

Fig. 6 Spectra of resolved energy $\mathbf{a}$, viscous $\mathbf{b}$, and magnetic $\mathbf{c}$ dissipation rates. Flows with $3 \mathrm{D}$ forcing, $R e=2,500, N=1$ and 5, and numerical resolution by $64 \times 64 \times 128$ functions are calculated using the dynamic model (solid lines), Smagorinsky model with constant $C_{S}$ (dashed line), and Smagorinsky model with constant $C_{S}$ adjusted according to (8) (dashed-dotted lines)

In order to test the formula (8) we conducted simple numerical experiments. Forced flows with $R e=2,500$ and numerical resolution $64 \times 64 \times 128$ were computed using the dynamic Smagorinsky model, simple Smagorinsky model with constant $C_{S}=C_{S 0}$, and the modification of the Smagorinsky model with constant $C_{S}$ adjusted at each time step according to (8). Each run started with the same isotropic initial velocity field. Calculations were performed for $N=1$ and $N=5$. After completion of transitional periods, the statistics were collected and time-averaged for the fully established anisotropic flows.

The results are presented in Fig. 6. One can see that the adjustment (8) results in almost exact reproduction of the spectra of energy and dissipation rates of flows obtained with the dynamic Smagorinsky model. On the contrary, the spectra calculated with the Smagorinsky model with constant $C_{S}$ are noticeably, albeit not very strongly, different, with typical over-dissipative suppression at large $k$.

It must be stressed that such an excellent agreement between the dynamic model and the model with adjusted $C_{S}$ is observed only for statistically steady periods of the flow evolution. The situation during the transient periods is quite different as illustrated by our test simulations of decaying turbulence. In the simulations, a fully developed forced non-magnetic flow is used as an initial conditions. At the moment $t=t_{0}$, the magnetic field is applied, and the forcing is discontinued, after which the flow is allowed to decay freely for several turnover times simultaneously developing anisotropy. Figure 7 shows the results of the dynamic LES of the process for $N=1$ and $N=5$. One can see in Fig. 7a, b that both $G$, which represents $C_{S} / C_{S 0}$ determined according to (8), and the dynamically determined $C_{S} / C_{S 0}$ decrease with time as the flow becomes anisotropic but at very different rates. Initially, the decay rate for the dynamic $C_{S}$ is almost zero, whereas the anisotropy coefficient drops rapidly. At later stages, $C_{S}$ decays faster than $G$ but remains significantly larger than predicted by (8). This is further illustrated in Fig. 7c. Similar delay in the modification of the Smagorinsky constant is demonstrated by the forced flows during the transitional stages of their development (see Fig. 1b, c).

One can be tempted by a simple explanation that the generation of dimensional anisotropy is an essentially linear process governed by the Joule dissipation (3). Its typical time scale is the Joule damping time $\tau \equiv \rho / \sigma B^{2}$. On the other hand, the variation of the Smagorinsky constant is associated with the nonlinear process of establishing new correlations between the turbulent rate of strain and stress tensors occurring at the time scale of the eddy turnover time $T=L / u$. One can expect slower evolution of $C_{S}$ at $N=T / \tau>1$. 

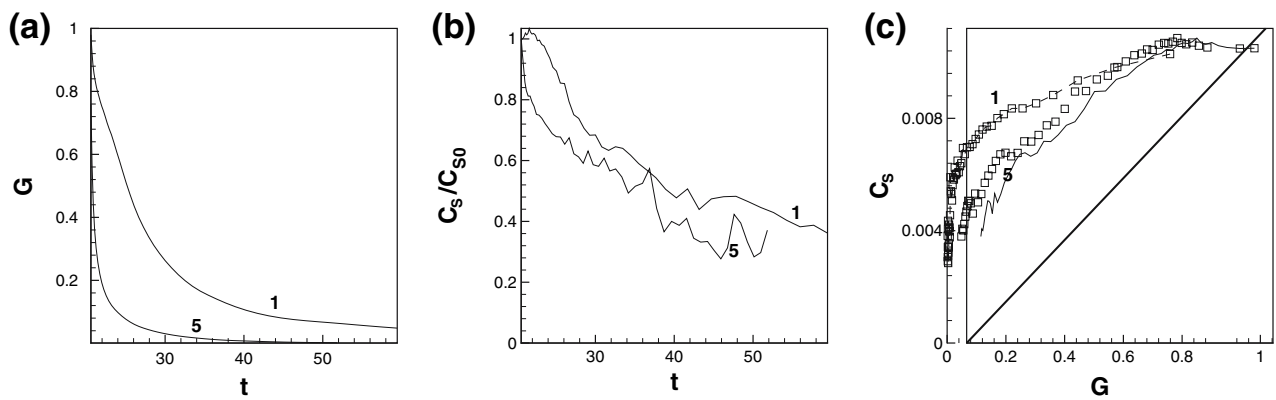

Fig. 7 Decaying turbulent flows with $R e=2,500, N=1$ and 5 calculated using the dynamic model. a Global anisotropy coefficient $G$, which also represents $C_{S} / C_{S 0}$ calculated according to the linear relation (8), b Smagorinsky constant $C_{S}$ scaled by the isotropic value $C_{S 0}, \mathbf{c}, C_{S}$ versus $G$

The explanation, albeit possibly relevant, is clearly an oversimplification. In particular, it does not explain the delay in the development of $C_{S}$ at $N=1$ (see Fig. 7).

The simulations of decaying homogeneous flows did not allow us to clearly separate between the results of the dynamic model, model with constant $C_{S}$ and model (8). The curves of $E(t), \epsilon(t)$, and $\mu(t)$ were found to be very close to each other. More definitive testing is required to identify the effect of the delay in development of $C_{S}$ on the accuracy of the model. This can be done in simulations of more realistic evolving flows, for example, of a free mixing layer.

\section{Concluding remarks}

We performed a series of LES of forced and decaying homogeneous MHD turbulence at low magnetic Reynolds number. Analyzing the flow properties in a wide range of the hydrodynamic Reynolds number and magnetic interaction parameter and at different large-scale dynamics and filter widths we found a clear confirmation that the effect of the dynamic adjustment of the Smagorinsky constant can be accurately approximated by a simple linear function of the global coefficient of gradient anisotropy.

Apart from purely theoretical interest, the relation has clear practical value. Calculation of $C_{S}$ in the dynamic model approximately doubles the amount of computations in comparison with the standard Smagorinsky model. ${ }^{1}$ This is undesirable in simulations of industrial processes such as, for example, the Czochralski growth of large crystals or continuous steel casting, the tasks computationally challenging even with the simplest turbulence models. With quantified dependence of $C_{S}$ on $G$ one could still use the standard model but avoid its over-dissipative character by adjusting $C_{S}$ to the strength of the flow anisotropy.

We found that the adjustment formula cannot be used in transient processes because development of anisotropy occurs at a faster rate than the modification of the Smagorinsky constant. Furthermore, the formula was obtained for homogeneous turbulence and may prove inaccurate in the presence of mean shear or rotation. It is possible that improved correlations can be developed for such situations but they are likely to be also more complex and less universal than our simple formula. Their advantage over the dynamic model is, thus, far from being obvious.

Acknowledgments The work is supported by the grant DE FG02 03 ER46062 from the U.S. Department of Energy. The authors are thankful to the referee for careful reading of the manuscript and useful comments.

\section{References}

1. Davidson, P.A.: An Introduction to Magnetohydrodynamics. Cambridge University Press, Cambridge (2001)

2. Moreau, R.: Magnetohydrodynamics. Kluwer, Dordrecht (1990)

3. Schumann, U.: Numerical simulation of the transition from three- to two-dimensional turbulence under a uniform magnetic field. J. Fluid Mech. 74, 31-58 (1976)

1 This may an overestimation for some, even statistically non-steady flows. The dynamic evaluation of $C_{S}$ is not always required at every time step. Accurate results are known to be obtained by evaluating $C_{S}$ once every few (sometimes as many as 10 ) steps. 
4. Alemany, A., Moreau, R., Sulem, P.L., Frisch, U.: Influence of an external magnetic field on homogeneous MHD turbulence. J. Mech. 18, 277-313 (1979)

5. Zikanov, O., Thess, A.: Direct numerical simulation of forced MHD turbulence at low magnetic Reynolds number. J. Fluid Mech. 358, 299-333 (1998)

6. Vorobev, A., Zikanov, O., Davidson, P.A., Knaepen, B.: Anisotropy of magnetohydrodynamic turbulence at low magnetic Reynolds number. Phys. Fluids 17, 125105 (2005)

7. Thess, A., Zikanov, O.: On the transition from two-dimensional to three-dimensional MHD turbulence. In: Proc. of 2004 CTR summer program. Stanford University, pp. 63-74 (2004)

8. Sommeria, J., Moreau, R.: Why, how, and when MHD turbulence becomes two-dimensional. J. Fluid Mech. 118, 507$518(1982)$

9. Hinze, J.O.: Turbulence. McGraw-Hill, New York (1959)

10. Knaepen, B., Moin, P.: Large-eddy simulation of conductive flows at low magnetic Reynolds number. Phys. Fluids 16, $1255(2004)$

11. Germano, M., Piomelli, U., Moin, P., Cabot, W.H.: A dynamic subgrid-scale eddy viscosity model. Phys. Fluids A 3,1760 (1991)

12. Lilly, D.K.: A proposed modification to the Germano subgridscale closure model. Phys. Fluids A 4, 633 (1992)

13. Pope, S.B.: Turbulent Flows. Cambridge University Press, Cambridge (2000)

14. Jiménez, J., Moser, R.D.: AIAA Paper 98-2891 (1998)

15. Pope, S.B.: Ten questions concerning the large-eddy simulation of turbulent flows. New J. Phys. 6, 35 (2004)

16. Zikanov, O., Vorobev, A., Thess, A., Davidson, P.A., Knaepen, B.: Anisotropy of MHD turbulence at low magnetic Reynolds number. In: Proc. of 2004 CTR summer program. Stanford University, pp. 35-46 (2004) 